\title{
MfbHLH38, a Myrothamnus flabellifolia bHLH transcription factor, confers tolerance to drought and salinity stresses in Arabidopsis
}

\author{
Jia-Rui Qiu', Zhuo Huang ${ }^{1 *}$ (D, Xiang-Ying Xiang ${ }^{1}$, Wen-Xin Xu ${ }^{1}$, Jia-Tong Wang ${ }^{1}$, Jia Chen ${ }^{1}$, Li Song ${ }^{1}$, Yao Xiao', \\ Xi Li', Jun Ma', Shi-Zhen Cai ${ }^{1}$, Ling-Xia Sun ${ }^{1}$ and Cai-Zhong Jiang ${ }^{2,3}$
}

\begin{abstract}
Background: The basic helix-loop-helix (bHLH) proteins, a large transcription factors family, are involved in plant growth and development, and defensive response to various environmental stresses. The resurrection plant Myrothamnus flabellifolia is known for its extremely strong drought tolerance, but few bHLHs taking part in abiotic stress response have been unveiled in M. flabellifolia.

Results: In the present research, we cloned and characterized a dehydration-inducible gene, MfbHLH38, from M. flabellifolia. The MfbHLH38 protein is localized in the nucleus, where it may act as a transcription factor. Heterologous expression of MfbHLH38 in Arabidopsis improved the tolerance to drought and salinity stresses, as determined by the studies on physiological indexes, such as contents of chlorophyll, malondialdehyde (MDA), proline (Pro), soluble protein, and soluble sugar, water loss rate of detached leaves, reactive oxygen species (ROS) accumulation, as well as antioxidant enzyme activities. Besides, MfbHLH38 overexpression increased the sensitivity of stomatal closure to mannitol and abscisic acid (ABA), improved ABA level under drought stress, and elevated the expression of genes associated with ABA biosynthesis and ABA responding, sucha as NCED3, P5CS, and RD29A.

Conclusions: Our results presented evidence that MfbHLH38 enhanced tolerance to drought and salinity stresses in Arabidopsis through increasing water retention ability, regulating osmotic balance, decreasing stress-induced oxidation damage, and possibly participated in ABA-dependent stress-responding pathway.
\end{abstract}

Keywords: bHLH transcription factor, Abiotic stress tolerance, Abscisic acid (ABA), Myrothamnus flabellifolia

\section{Background}

Plants, as sessile species, are vulnerable to changing environmental conditions, and the increasing drought and salinity stresses usually restrict the development and growth of plants through disturbing ion homeostasis, reducing nutrient uptake, and exacerbating oxidation

\footnotetext{
* Correspondence: huangzhuo@sicau.edu.cn

${ }^{1}$ College of Landscape Architecture, Sichuan Agricultural University, Wenjiang 611130, Sichuan, China

Full list of author information is available at the end of the article
}

stress [1]. To adapt to these disadvantaged conditions of environmental stresses, plants have formed a variety of complex coping mechanisms during the evolution process. Signal transduction and transcription regulation play important roles in the sophisticated biochemistry and molecular regulatory networks when plants replying to different stresses. Abscisic acid (ABA) as a ubiquitous plant hormone is involved in the network of stress signaling responding to environmental stimulation and plays an irreplaceable part in diverse biological processes of plants

(c) The Author(s). 2020 Open Access This article is licensed under a Creative Commons Attribution 4.0 International License, which permits use, sharing, adaptation, distribution and reproduction in any medium or format, as long as you give appropriate credit to the original author(s) and the source, provide a link to the Creative Commons licence, and indicate if changes were made. The images or other third party material in this article are included in the article's Creative Commons licence, unless indicated otherwise in a credit line to the material. If material is not included in the article's Creative Commons licence and your intended use is not permitted by statutory regulation or exceeds the permitted use, you will need to obtain permission directly from the copyright holder. To view a copy of this licence, visit http://creativecommons.org/licenses/by/4.0/ The Creative Commons Public Domain Dedication waiver (http://creativecommons.org/publicdomain/zero/1.0/) applies to the data made available in this article, unless otherwise stated in a credit line to the data. 
under biotic and abiotic stress conditions [2, 3]. Elevated ABA levels can activate certain transcription factors (TFs), thereby regulating the expression of diverse downstream genes [4]. Hitherto, a range of stress-responsive TFs have been reported with regard to resisting abiotic stress tolerance in different plants $[5,6]$.

In the plant kingdom, the basic helix-loop-helix (bHLH) transcription factors belong to a large superfamily. It could be subdivided into 26 subsections and participates in multiple transcriptional regulatory pathways [7]. The first bHLH transcription factor, regulatory gene $R$, was isolated from maize, and there are 167 and 177 members in Arabidopsis and rice, respectively [8-10]. The bHLH transcription factors are characterized by a highly conserved bHLH domain, comprising of a basic region at its $\mathrm{N}$-terminus and an HLH region following closely. These two regions function as DNA binding and promoting protein-protein interactions, respectively $[11,12]$. The core DNA sequence element of target genes recognized by the bHLH proteins is a consensus motif called the E-box $\left(5^{\prime}\right.$-CANN TG-3'), with the palindromic G-box (5'-CACGTG-3') being one of the most common forms [13, 14].

In recent years, increasing evidence was found that bHLHs are involved in multiple abiotic stress responses by the ABA signal transduction pathway in plants. AtbHLH92 [15], AtbHLH17 (AtAIB) [16, 17], and AtbHLH122 [16] regulated response to drought, salinity, osmotic, oxidative or cold stress through an ABAdependent pathway. Grape $V v b H L H 1$ endowed transgenic Arabidopsis with improved tolerance to salinity and drought via ABA signal network [18]. Chrysanthemum CmbHLH1 can promote iron absorption through upregulating the expression of Fe-deficiency-responsive genes, and in which ABA may play a key role [19].

bHLH proteins of group Ib, such as bHLH38, bHLH39, bHLH100, and bHLH101, are well known to be involved in regulating iron homeostasis by interacted with FE-DEFI CIENCY INDUCED TRANSCRIPTION FACTOR (FIT) (bHLH29), and their expression is strongly induced by iron starvation [20]. They could be regulated by AtMYC2 [20], which functions as a transcriptional activator in ABA-inducible gene expression under drought stress in Arabidopsis [21]. The ABRE element (abscisic acid response element), usually found in the promoter region of ABA-inducible genes, was detected in the promoters of the co-expressed genes AtbHLHO39 and AtbHLH101 [22]. Kurt and Filiz also found ABRE elements in the promoter region of bHLH38/39/100/101 gene in Arabidopsis, rice, soybean, tomato, and maize [23]. These results suggested that these group Ib bHLH proteins may participate in ABA-response pathways. However, it is still unclear if they take part in response to other abiotic stresses, such as drought and salinity.
Woody resurrection plant Myrothamnus flabellifolia Welw. is a unique dwarf shrub worldwide and grows in poor rock conditions [24, 25]. A long period of evolution, as well as mighty adaptability to extreme drought surroundings, make M. flabellifolia develop a powerful survival strategy including a well-developed root system and the capability to recover from dehydration [26-28]. Based on the previous study, there are a variety of TFs that play a part in the transcriptional regulatory networks during the dehydration process in M. flabellifolia, in which $M f b H L H 38$ was obviously up-regulated in initial period of dehydrating treatment [29]. The strong stress resistance mechanism of the resurrection plant is inseparable from whose multiple adversity genes. In this study, the MfbHLH38 was cloned from M. flabellifolia. The sequence analysis and functional characterization were performed. Its roles to enhance drought and salinity tolerance were determined and the underlying mechanisms were preliminarily investigated and discussed.

\section{Results}

\section{Cloning and sequence analysis of $M f b H L H 38$}

Using PCR amplification, the cDNA sequence of MfbHLH38 was obtained from M. flabellifolia. The obtained sequence is $720 \mathrm{bp}$ in length and encodes an putative protein of 239 amino acids. The theoretical isoelectric point of $\mathrm{MfbHLH} 38$ is 8.84 and the predicted molecular mass is $27.15 \mathrm{kDa}$. In silico predication detected a a typical bHLH domain and putative bipartite nuclear localization signal (NLS) of "KKLNHNASERDRRKKIN" (Fig. 1a). Multiple alignment of deduced amino acids of MfbHLH38 and several highly homologous bHLH proteins showed a conserved basic region followed by an HLH domain (Fig. 1a). We further performed phylogenetic analysis and found that the MfbHLH38 was most homologous to VvORG2, PtORG2, HbORG2-like, JcORG2 (ORG2 also named bHLH38), and AtbHLH38 which are from Vitis vinifera, Populus trichocarpa, Hevea brasiliensis, Jatropha curcas, and Arabidopsis thaliana, respectively. (Fig. 1b).

\section{MfbHLH38 is localized in the nucleus}

The predicted NLS in MfbHLH38 (Fig. 1a) suggested that it may function in the nucleus. To confirm this speculation, we performed transient expression of the 35S::MfbHLH38-YFP into leaf epidermal cells of tobacco. Observation using confocal microscope showed that the fluorescence could be detected in the whole cell of 35S::YFP, whereas the intense yellow fluorescence was specifically appeared in the nucleus of 35S:: MfbHLH38YFP transformed cell. These results proved that MfbHLH38 is located in the nucleus (Fig. 2). 
a

AtbHLH38
PdbHLH
PpORG2
PbbHLH
VvORG2
MfbHLH38

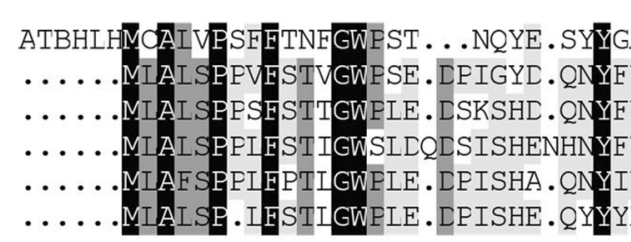

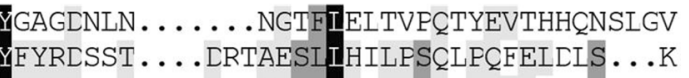
YRLSET .... ECIAESEIRILPSCLECVEFARSTEST YFYSKDITTTNLECTADSETHLLPSHHFKVDILRSTFST bipartite NLS

AtbHLH38

-

PdbHLH

PpORG2

PbbHLH

VvORG2

MfbHLH38

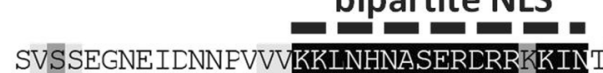

YGETET . . . . . . SESFIH.LSSSQPCVEINCS . . . .

TISGL . . . NSSGSTVAKKLNHNASERLRRKKIN
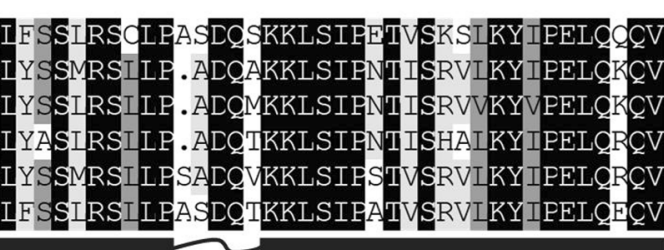

TASGE . . . YSGVSTMVKKLNHNASERLRROKIN

TVTGN . . . YSSVCPMAKKLNHNASERLRRKKII

TPSAA . . V.VSGNPTMVKKLNHNASERLRRIKIN

TVSGT . . . ITGGCূTIVKKLNHNASERLRRKKINS

IKKLSIP
basic
Helix
Loop
Helix

AtbHLH38

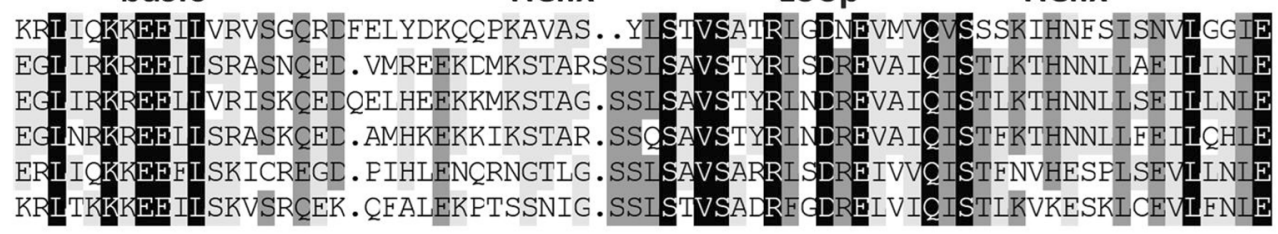

PdbHLH

PpORG2

PbbHLH

VvORG2

MfbHLH38

KRITKKKEEII SKVSRCEK. CEALEKFTSSNIG . SSI STVSADR GDREIVIQISTIKVKESKICEVIFNIE

AtbHLH38

PdbHLH

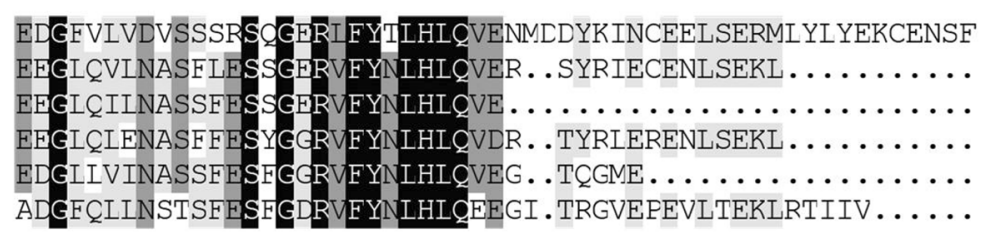

PpORG2

PbbHLH

VvORG2

MfbHLH38

b

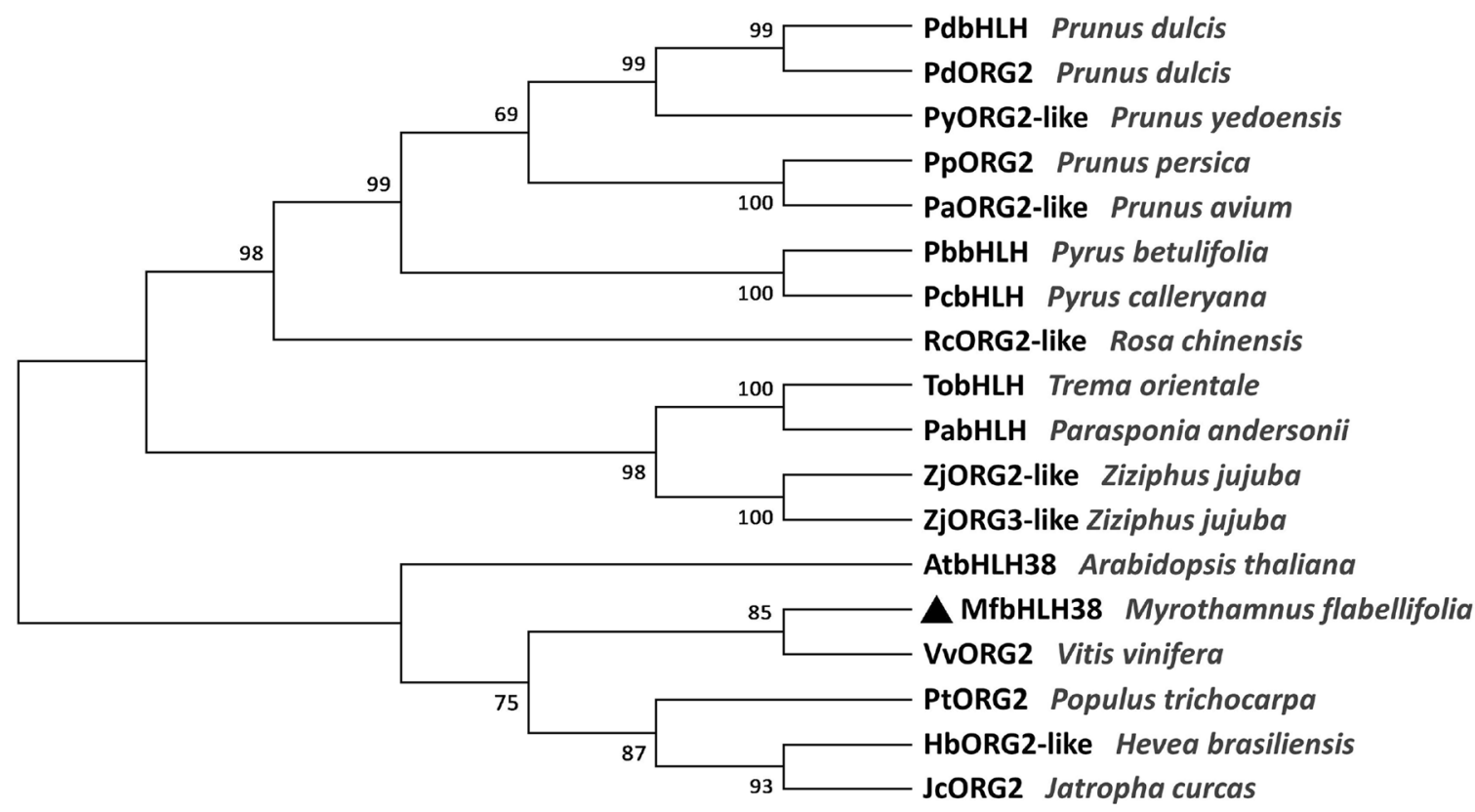

Fig. 1 (See legend on next page.) 
(See figure on previous page.)

Fig. 1 Multiple sequence alignment (a) and phylogenetic analysis (b) of MfbHLH38 and several highly homologous bHLH proteins. Black and gray shade showed identical and similar amino acids, respectively. Amino acids marked by the dashed line was the deduced NLS. The basic region was marked by the white box, and the curve-linked black boxes indicated the conserved HLH domain. Phylogenetic reconstruction using the neighbor-joining method. The accession numbers for the sequences used are as follows: AtbHLH38 (AT3G56970.1) from Arabidopsis thaliana; VVORG2 (RVW89141.1) from Vitis vinifera; PdbHLH (BBH07182.1) from Prunus dulcis; PpORG2 (XP_020423445.1) from Prunus persica; PyORG2-like (PQM37255.1) from Prunus yedoensis var. nudiflora; PaORG2-like (XP_021822000.1) from Prunus avium; TobHLH (PON88894.1) from Trema orientale; PbbHLH (AMX27896.1) from Pyrus betulifolia; PcbHLH (AMX27897.1) from Pyrus calleryana; ZjORG2-like (XP_024922954.1) from Ziziphus jujuba; HbORG2-like (XP_021669234.1) from Hevea brasiliensis; RcORG2-like (XP_024162921.1) from Rosa chinensis; PabHLH (PON38640.1) from Parasponia andersonii; ZjORG3-like (XP_015900576.1) from Ziziphus jujuba; PtORG2 (XP_002307969.3) from Populus trichocarpa; JcORG2 (XP_012072671.1) from Jatropha curcas; and PdORG2 (BBH07187.1) from Prunus dulcis

\section{Overexpressing MfbHLH38 increased drought and salt tolerance}

To analyze the potential roles in response to abiotic stress, the MfbHLH38 was introduced into Arabidopsis driven by $35 \mathrm{~S}$ promotors. $\mathrm{T}_{1}$ transgenic Arabidopsis lines that overexpressing the MfbHLH38 gene was acquired from kanamycin resistance screening, and three homozygous $T_{3}$ transgenic lines were randomly selected and used for further analysis. The qRT-PCR analysis indicated that that expression level of MfbHLH38 could be detected in all three selected transgenic lines, in which Line D exhibited significantly higher expression level than the other two lines (Fig. 3A).

In order to verify whether $M f b H L H 38$ is associated with drought and salinity stress tolerance, WT and transgenic lines were subjected to stress treatments at both the seedling and adult stages. At the seedling stage, there was no significant difference between wild type and transgenic plants under normal conditions (Fig. 3B). Under treatments of mannitol and salt, transgenic lines exhibited significantly longer roots. This difference was more obvious when rather moderate concentrations of mannitol $(250 \mathrm{mM})$ and $\mathrm{NaCl}(100 \mathrm{mM})$ were used (Fig. 3B, C, and D). Consistently, larger leaf area was also found in transgenic lines compared with WT (Fig. 3B).

At the adult stage, treatments of natural drought and $300 \mathrm{mM} \mathrm{NaCl}$ were performed on four week old transgenic and WT plants growing in soil. The morphological difference was not remarkable among the transgenic and WT plants before and at early stages of two treatments (Fig. 4). Withholding watering (DAW) for 10 days, the wilting degree of the WT leaves was significantly higher than that of the transgenic lines, and the leaf chlorophyll content of later was 1.48-1.58 times higher than that of former (Fig. 4a, c). At $15 \mathrm{DAW}$, the WT leaves were basically withered, while a considerable number of leaves on transgenic plants remained light green (Fig. 4a). Three days after re-watering, transgenic plants were partially restored, however, the almost all of the WT plants were dead (Fig. 4a).

The salinity stress negatively influence the plant growth, which was visible about seven days of salt treatment (Fig. 4b), and the leaf chlorophyll content of MfbHLH38 transgenic lines was 1.20-1.26 times higher than that of WT (Fig. 4c). Twelve days after exposure to

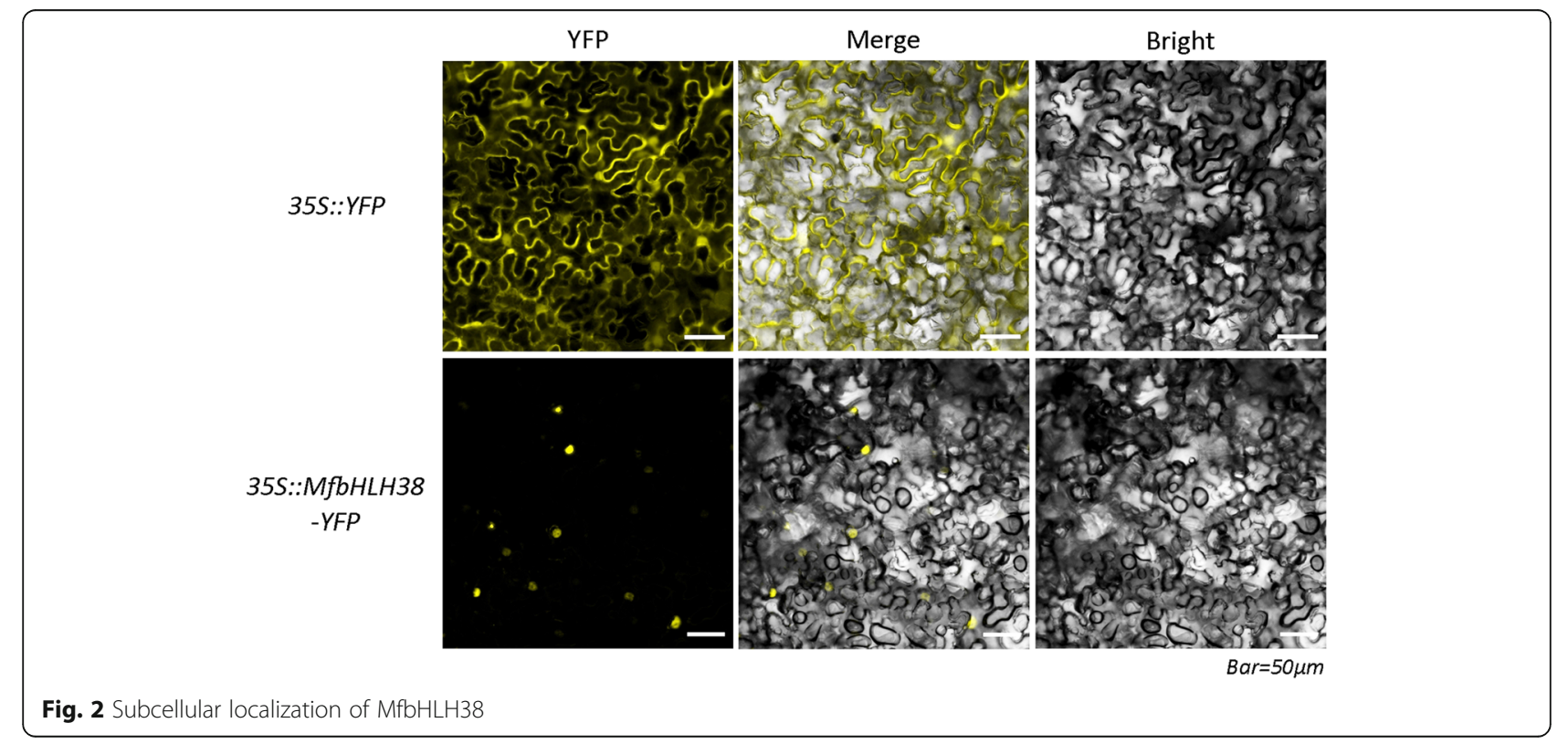




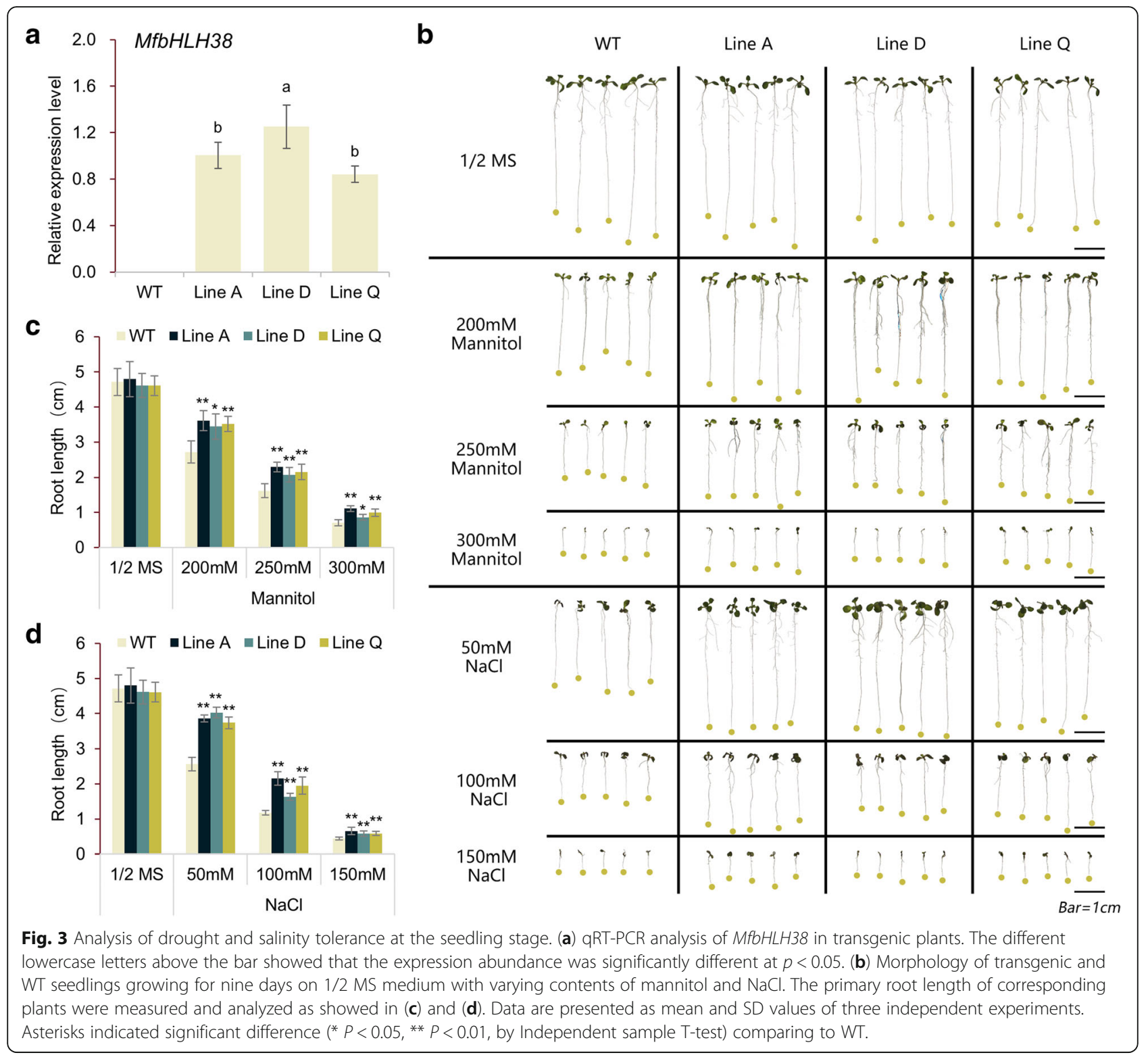

salinity stress, more withered leaves were appeared on WT plants comparing to those of transgenic lines. After 18 days, approximately more than $1 / 3$ of transgenic plants stayed green and flowered, whereas almost all leaves of WT were withered (Fig. 4b).

The dynamic water loss rate (WLR) of detached leaves were measured during dehydration. As shown in Fig. 4d, WLR of transgenic plants were significantly lower than that of WT at all-time points except for $0 \mathrm{~h}$, indicating that overexpression of $M f b H L H 38$ slowed down the water loss. Malondialdehyde (MDA) can severely damage plant cell membranes, thus degree of MDA accumulation is usually considered as a indicator of membrane-lipid peroxidation. In our experiment, although both the drought and salt treatments elevated the MDA content in either the WT or transgenic plants, it stayed in significantly lower levels than that of WT (Fig. 4e).

We compared the contents of several osmotic adjustment substances, including proline, soluble protein, and soluble sugar, among the transgenic and WT plants before and after treatments. Our results revealed the similar patterns that both the drought and salt stresses enhanced osmolyte accumulation in WT and the transgenic lines, but those in later was significantly higher that that of the former (Fig. 4f-h).

\section{Effect of $M f b H L H 38$ overexpression on antioxidant metabolism}

It is well known that rising of lipid peroxide will aggravate the cellular oxidative damage when plants suffer from the abiotic stresses. This is caused by excessive accumulation 


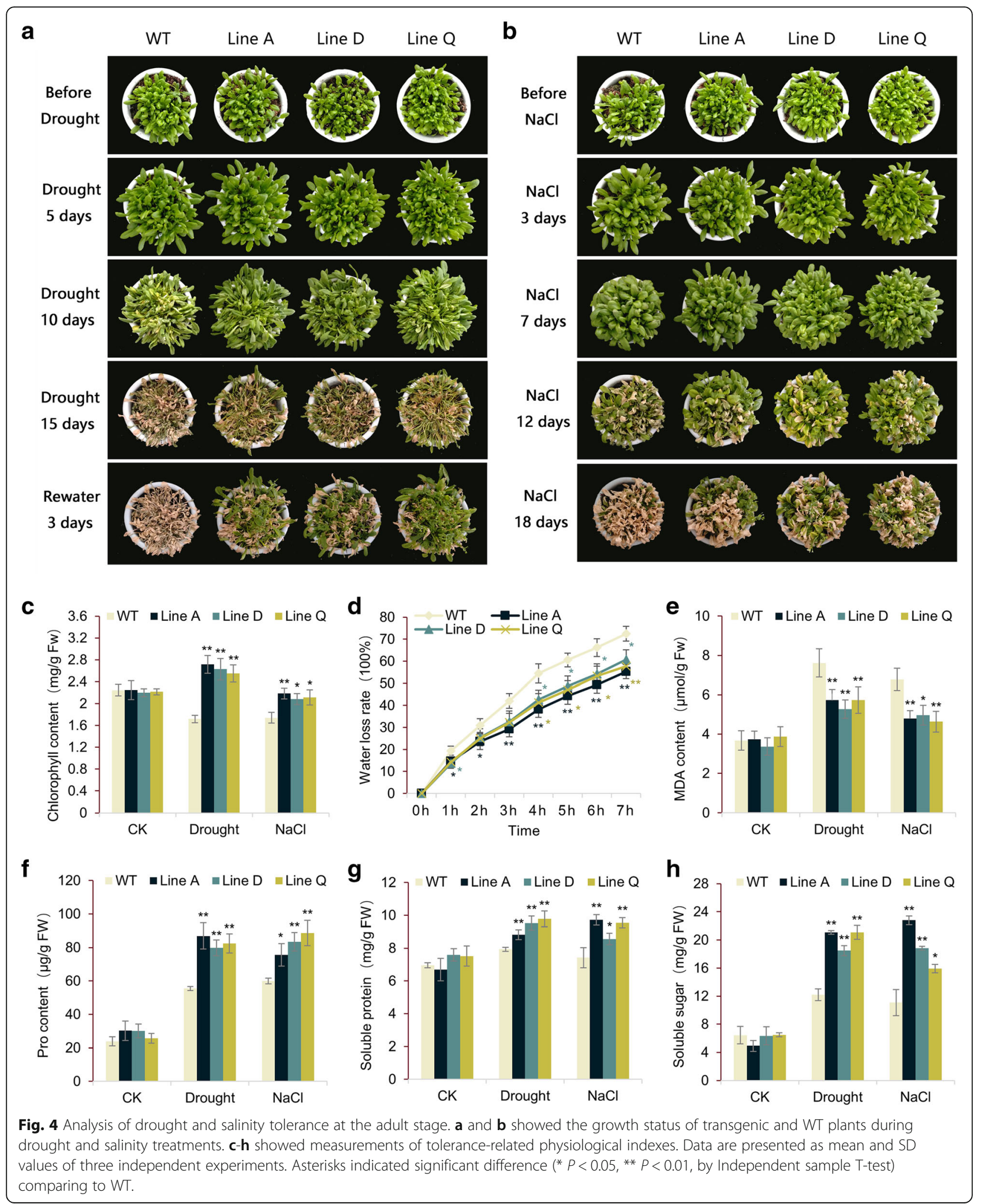


of ROS (reactive oxidative species), such as hydrogen peroxide $\left(\mathrm{H}_{2} \mathrm{O}_{2}\right)$ and superoxide anion radical $\left(\mathrm{O}_{2}{ }^{-}\right)$. We employed histochemical staining by $3,3^{\prime}$-diaminobenzidine (DAB) and nitroblue tetrazolium (NBT) to evaluate cellular ROS content after drought and salt treatments. The WT leaf could be stained in darker color and larger area comparing to those of transgenic plants (Fig. 5a and b), indicating slighter cellular oxidative damage occurred in the transgenic lines. Consistently, less $\mathrm{H}_{2} \mathrm{O}_{2}$ content and higher anti-superoxide anion activity were detected in three transgenic lines (Fig. $5 \mathrm{c}$ and d).

Furthermore, we measured activities of superoxide dismutase (SOD), peroxidase (POD), and catalase (CAT), which are the key enzymes participating in ROS scavenging. Along with the ROS level increasing, the activities of SOD, POD, and CAT were significantly elevated by drought and salt treatments in both the WT and the transgenic plants. However, the enzyme activities of transgenic plants are apparently higher than that of WT (Fig. 6e-g), showing that overexpression of MfbHLH38 could decrease cellular oxidative damage under stressful conditions through increasing ROS scavenging capacities.

\section{MfbHLH38 promoted stomatal closure and the biosynthesis of endogenous ABA}

The ABA-mediated stomatal movement plays a central role in transpiration upon water stress. We assessed the stomatal closure under $300 \mathrm{mM}$ mannitol and $20 \mu \mathrm{M}$ ABA treatments. For both WT and transgenic lines, most of the stomata were open under normal conditions (Fig. 6a), and the stomatal aperture (width/length ratio) of was significantly different between transgenic and WT plants (Fig. 6b). Treatments of mannitol and ABA reduced stomatal aperture of three transgenic lines to $0.23-0.25$ and $0.09-0.10$, respectively, which were remarkably lower than those of WT plants $(0.32$ and 0.21$)$ (Fig. 6b). These results demonstrated that MfbHLH38 promoted stomatal closure in response to mannitol and $\mathrm{ABA}$. We also measured $\mathrm{ABA}$ content under drought stress. The accumulation of ABA in WT and transgenic lines increased significantly after drought treatment, however, $\mathrm{ABA}$ contents in three transgenic lines were 1.48-2.18 times higher than that of WT plants (Fig. 6c). This result indicated the overexpression of $\mathrm{MfbHLH} 38$ promoted ABA synthesis under drought.

\section{Overexpression of $\mathrm{MfbH} \mathrm{HH} 38$ up-regulated expression levels of ABA-responsive genes}

To further explorer the potential molecular mechanisms underlying enhanced drought and salinity tolerance by $M f b H L H 38$-overexpressing, we measured the expression levels of three stress-induced and ABA-responsive genes under artificially simulated drought treatment $(10 \%$ PEG-6000) and salt treatment (300 $\mathrm{mM} \mathrm{NaCl)} \mathrm{for} \mathrm{one}$ day and four days, respectively. As shown in Fig. 7a, the similar expression level of NCED3 were found among WT and transgenic lines before treatments. After treatments, the expression levels in all three transgenic lines increased faster and higher than that of WT. The P5CS and $R D 29 A$ exhibited slightly higher expression levels in transgenic lines before treatments (Fig. 7b and c). Under drought treatment, the expression levels of P5CS and $R D 29 A$ in $M f b H L H 38$-overexpressing lines were remarkably increased and significantly higher than those of WT plants. Under salinity stress, expression levels of P5CS and RD29A showed a similar trend of NCED3. All these results suggested that $M f b H L H 38$ positively regulated the expression of ABA-responsive genes in Arabidopsis directly or indirectly.

\section{Discussion}

Stress tolerance of plant depends on the adversity genes, and overexpression of these genes can improve the plant's ability to adapt to a variety of environmental stresses [30]. The function of bHLH38 in stress tolerance has not yet been exploited, even though its role in maintaining cellular iron homeostasis has been extensively investigated. In the present study, we isolated and identified MfbHLH38 from M. flabellifolia. It contains the high conservative bHLH domain (Fig. 1a), and showed high homology with VvORG2 (Vitis vinifera), PtORG2 (Populus trichocarpa), HbORG2-like (Hevea brasiliensis), and JcORG2 (Jatropha curcas) (Fig. 1b).

Further investigation showed that overexpressing MfbHLH38 in Arabidopsis endows it with tolerance to drought and salinity stresses, as revealed by better growth vigor of $M f b H L H 38$-overexpressing plants under stress treatments and at either seedling or adult stages (Fig. 3b, 4a and b). The increased adaptability of these transgenic plants to drought and salinity stresses was inseparable from the simultaneous adaptive changes in external morphology and biochemical levels.

Plants can draw water from deep soil through welldeveloped root systems, thereby improving the efficiency of limited water use under drought conditions [31]. In seedling stress assays, MfbHLH38 transgenic lines showed stronger growth and longer main roots (Fig. 3c and d). Additionally, plants can reduce water loss in drought conditions by promoting stomatal closure, and ABA-regulated stomatal movement enables plants to improve water retention. For example, stomatal aperture significantly decreased in PebHLH35-overexpressing Arabidopsis with the increase of drought degree, showing that PebHLH35 overexpression exhibited good tolerance in transgenic plants to drought stress [32]. In this study, stomatal movements of MfbHLH38 overexpression lines were more sensitive to mannitol treatment and exogenous ABA (Fig. 6a, b), and exhibited significantly lower water loss rate (Fig. $4 \mathrm{~d}$ ). These 

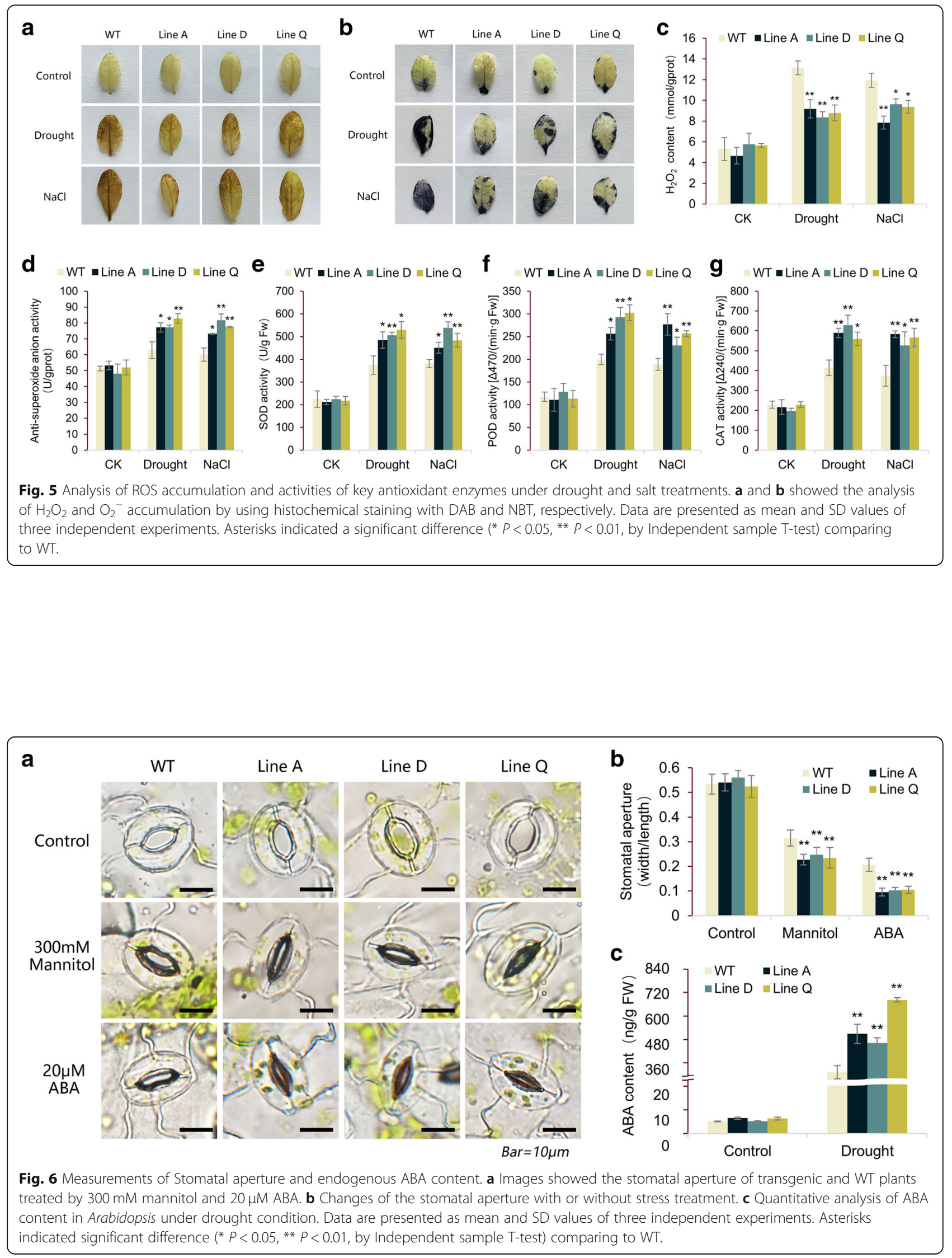


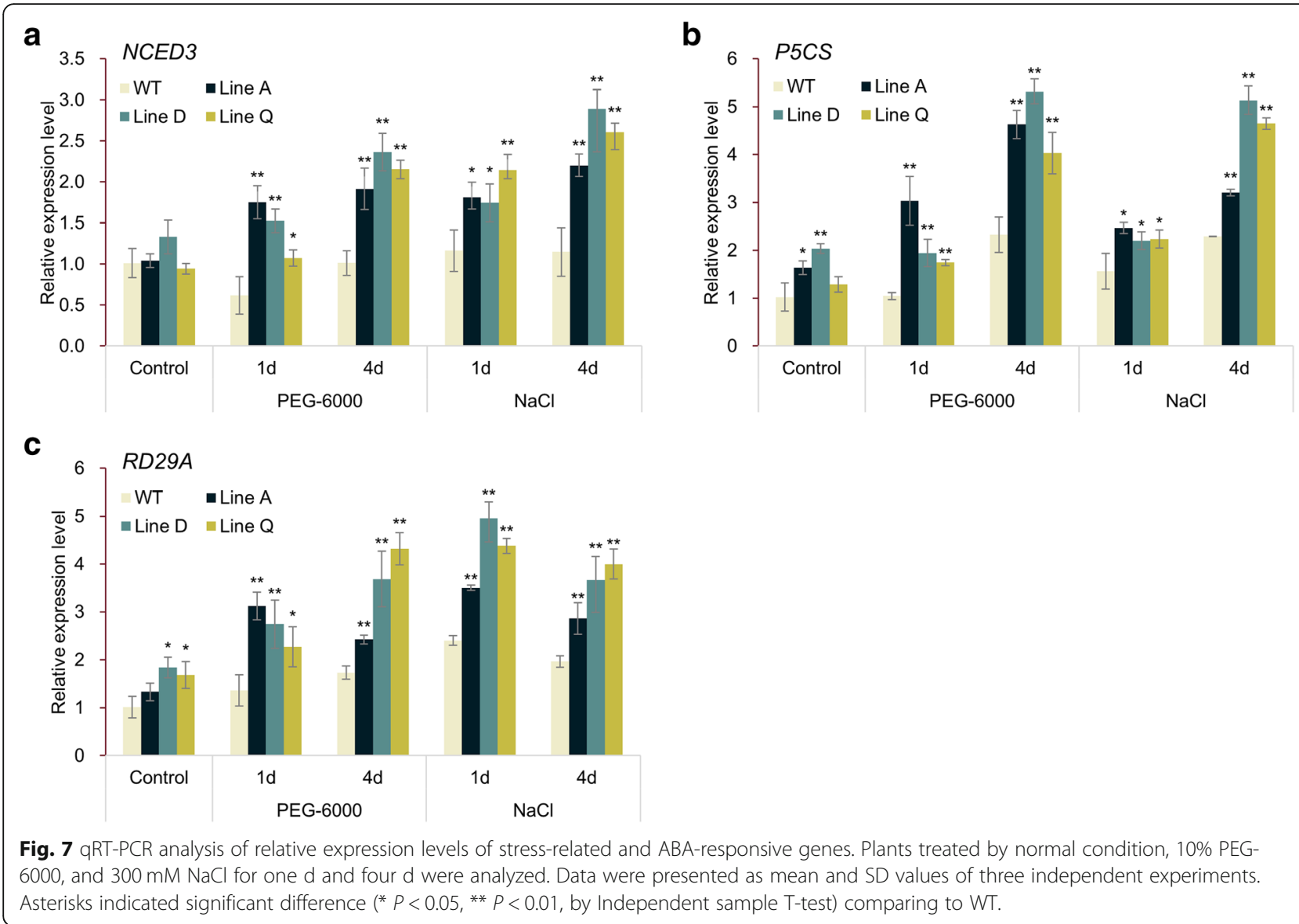

results showed that $M f b H L H 38$ could enhance water uptake and retention, and therefore provides a better water use condition under stress treatments.

Plants can generate osmoregulation substances including proline, soluble proteins as well as soluble sugars under stresses. The accumulations of these osmotic modifiers could assist plants to resist the environmental stresses via maintaining the osmotic equilibrium [33, 34]. Yang et al. found that after drought and salinity stress treatments, the content of proline and soluble sugar in TabHLH1 transgenic Arabidopsis increased, and was significantly higher than that of WT plants [35]. Our data showed that the contents of these three osmoregulation substances were increased obviously upon drought and salinity stresses (Fig. 4f-h). This result was also supported by higher expression levels of P5CS under stress in three transgenic lines comparing to WT plants. Thus, MfbHLH38 may be involved in osmotic regulation directly or indirectly.

MDA content is a measurement of the degree of oxidative stress via reflecting plant membrane-lipid peroxidation level [36]. In this study, less MDA accumulation was detected in $M f b H L H 38$ transgenic lines (Fig. 4e), suggesting that overexpression of MfbHLH38 could maintain the membrane-lipid structure stable under drought and salinity conditions. ROS accumulation in plant cells, such as hydrogen peroxide $\left(\mathrm{H}_{2} \mathrm{O}_{2}\right)$ and superoxide anion $\left(\mathrm{O}_{2}{ }^{-}\right)$, promoted by drought and salt stresses leads to irreversible damages to plants [37, 38]. As shown by the results of histochemistry staining with DAB and NBT, and measurements of $\mathrm{H}_{2} \mathrm{O}_{2}$ content and anti-superoxide anion activity, MfbHLH38 transgenic lines presented a less increase of ROS accumulation under drought and salinity stresses (Fig. 5a-d). Antioxidant enzymes have important functions in reducing oxidative injury to plants caused by drought and salt stresses [39]. Consistent with lower ROS accumulation, overexpression of $M f b H L H 38$ remarkably increased the activities of antioxidant enzymes, SOD, CAT, and POD, upon stress conditions (Fig. 5e-g), indicating that MfbHLH38 could enhance the ROS scavenging system and reduced oxidative injury under stress.

As a critical plant hormone, ABA is involved in various developmental processes and stress signaling transduction mechanisms in plants [40]. Several bHLH TFs were reported to induce ABA biosynthesis and are involved in stress tolerance. For example, the grape VvbHLH1 conferred great tolerance for transgenic Arabidopsis to drought stress by increasing ABA levels [18]. 
In the present study, the significantly higher ABA contents in three transgenic lines comparing to WT under drought treatment were found (Fig. 6c). This is evident that overexpression of $\mathrm{MfbHLH} 38$ enhanced ABA biosynthesis, which explained promoted stomatal closure under stress treatments, as well as elevated expression levels of ABA-biosynthesis gene NCED3 and ABA- and stress-responsive genes P5CS and RD29A (Fig. 7). These results suggested that $M f b H L H 38$ might positively function in plant defense via the ABA-dependent pathway.

bHLH38 plays a positive regulatory role in iron deficiency [41]. In this study, we demonstrated that MfbHLH38 also positively regulated drought and salt stress responses. This result suggested that $\mathrm{M} f b H L H 38$ might mediate crosstalk between regulating $\mathrm{Fe}$ deficiency and other abiotic stress responses [42]. As iron acts on the particular active sites of some antioxidant enzymes, for instance, SOD, POD, and CAT [43], overexpressing $\mathrm{M} f b H L H 38$ may help to retain or increase activities of antioxidant enzymes through promoting Fe uptake. Furthermore, iron (Fe) plays an important role in chlorophyll biosynthesis and photosynthesis. However, water deficiency affects transporting nutrients to roots and nutrients utilization ratio, such as micronutrient $\mathrm{Fe}[44,45]$. And high-concentration sodium also interferes with the uptake and translocation of iron and other mineral elements $[46,47]$. Then the stresses initiated Fe deficiency can further cause the damage on chlorophyll, thereby seriously interfere with the photosynthesis process it involved [48]. Babaeian et al. applied Fe fertilizers to the leaves of sunflowers at the flowering and seed filling stages under drought conditions, and higher chlorophyll fluorescence (FV/FM) and chlorophyll content compared to the control plants were detected, proving that the $\mathrm{Fe}$ helps to improve photosynthesis in drought stress [49]. Consequently, it is possible that overexpression of $\mathrm{MfbHLH} 38$ may promote Fe uptake under stressful condition and slow down the damage on chlorophyll and its biosynthesis, and hence ensure plants better growth comparing to WT. However, further study is deserved to excavate the exact mechanisms underlying the positive role of MfbHLH38 in regulating abiotic stress response.

\section{Conclusions}

This study reported the characterization of MfbHLH38 encoding a bHLH transcription factor homologous to AtbHLH38. We demonstrated that heterologous expression of $M f b H L H 38$ in Arabidopsis significantly enhanced tolerance to drought and salinity by increasing water retention ability, regulating osmotic balance, strengthening stressinduced oxidation scavenging system, and possibly participated in ABA-dependent stress-responding pathway. This is the first report of involvement of bHLH38 in drought and salinity stress tolerance regulation. MfbHLH38 may have potential to be utilized in drought and salt stress tolerance improvement in plants. And it will be beneficial to further explore the molecular mechanism underlying the survival of M. flabellifoli from the desiccation environment.

\section{Methods \\ Plant materials and growth conditions}

The M. flabellifolia was originally obtained from Dr. Matthew Opel (University

of Connecticut). M. flabellifolia was firstly recorded and named by Welwitsch [50]. One of the voucher specimens of this species could be found in National $\mathrm{Mu}$ seum of Natural History (10th St. \& Constitution Ave. NW Washington, D.C. USA) at Botany Collections (US Catalog No.: 2921412 Barcode: 00072109). The plants used in this study were grown in plastic pots under condition of $12 \mathrm{~h}$ light $/ 12 \mathrm{~h}$ dark at $22^{\circ} \mathrm{C} / 18^{\circ} \mathrm{C}, 60 \%$ relative air humidity and sufficient light .

Seeds of Arabidopsis ecotype Columbia (Wild-type, WT) and overexpression lines were sterilized using diluted bleach solution for $5 \mathrm{~min}$, and washed with using sterilized deionized water for three times. The sterilized seeds were placed on 1/2-strength Murashige and Skoog (MS) medium containing $0.7 \%(\mathrm{w} / \mathrm{v})$ agar and $2 \%(\mathrm{w} / \mathrm{v})$ sucrose and with adjusted $\mathrm{pH}$ of 5.8-6.0. After vernalizationat $\left(4{ }^{\circ} \mathrm{C}\right.$ for two days), the medium plate was placed in an illuminating incubator for about 10 days. The young seedlings were transplanted into pots filled with cultivation substrate of soil and vermiculite (1:1) in a growth chamber, and then were grown under the long day $\left(16 \mathrm{~h} \mathrm{light} / 8 \mathrm{~h}\right.$ dark and $\left.24^{\circ} \mathrm{C} / 22^{\circ} \mathrm{C}\right)$ condition and about $75 \%$ relative humidity for four weeks before stress treatments.

\section{Cloning and sequence analysis of $M f b H L H 38$}

Total RNA was extracted from fresh leaves using Plant Total RNA Isolation Kit (TINAGENE Co., Beijing, China). The first-strand cDNA synthesis was conducted using Reverse Transcriptase M-MLV (RNaseH-) (Takara Bio, Dalian, China) according to the protocol provided by the kit. The coding sequence (CDS) of MfbHLH38 was amplified by PCR using Phanta Max Super-Fidelity DNA Polymerase (Vazyme Biotech Co., Nanjing, China) with a primer pair, 5'-TCCCCCGGG ATGCTAGCTC TATCTCCTTT-3' (SmaI site is underlined) and $5^{\prime}$ GACTAGTTCATACGATGATGGTACGTA-3' (SpeI site is underlined). The target amplified fragment was recovered from gel and ligated onto the pEasy-T1 Simple vector (TransGen Biotech, Beijing, China). The resulting construct, of pEasy-T1-MfbHLH38, was transformed into E. coli strain $\mathrm{DH} 5 \alpha$ and the putative positive clones were confirmed by Sanger sequencing (TsingKe Biotech Co., Beijing, China). 
The open reading frame (ORF) was detected by ORFfinder of NCBI (https://www.ncbi.nlm.nih.gov/orffinder/ ). Isoelectric point (pI) and molecular weight were caculated by ExPASy (https://web.expasy.org/compute_pi/). SMART (http://smart.embl-heidelberg.de/) was used to analyzing potential conserved domains in the deduced protein sequence. Homologs of MfbHLH38 from different species were searched using BLASTP (https://blast. ncbi.nlm.nih.gov/Blast.cgi) against nr database. Multiple alignments and subsequent phylogenetic analyses were performed with MEGA 7.0 [51] using the neighborjoining method with the bootstrap test of 1000 replicates. The secondary structure of deduced protein was predicted by Jpred 4 (http://www.compbio.dundee.ac.uk/ jpred/index.html).

\section{Subcellular localization of MfbHLH38}

The complete ORF without the termination codons of MfbHLH38 was obtained with primers containing adaptor sequences (italicized) complementary with vector sequence flanking ligation site, forward, 5'- ACCAGTCTCTCTCT CAAGCTTATGCTAGCTCTACTATCTCCTTT - 3' (Hin$d$ III site is underlined) and reverse, 5'- GCTCACCATA CTAGTGGATCCTACGATGATGATGGTACGTA - 3'

(BamHI site is underlined). The amplified fragment was double-digested by BamHI and HindIII and inserted into the pHB-YFP vector to formed a expression construct MfbHLH38-YFP, which was fused with the gene encoding yellow fluorescent protein (YFP) driven by a CaMV (Cauliflower Mosaic virus) 35S promoter. Both the 35S:: MfbHLH38-YFP and 35S::YFP were transformed into the Agrobacterium tumefaciens strain GV3101 by employing the freezing-thawing method, respectively. Leaves of four-weekold (Nicotiana benthamiana) wild-type tobacco were injected with $A$. tumefaciens. All the transformed tobacco plant were grown in dark for $16 \mathrm{~h}$ at $22^{\circ} \mathrm{C}$ and then moved to normal condition for two days before observing the YFP by a laser confocal scanning microscope (Nikon, Tokyo, Japan).

\section{Plasmid construction transformation in Arabidopsis}

The complete coding region of $M f b H L H 38$ was amplified using gene-specific primers supplied with either a SmaI or SpeI restriction site. The amplicon was double digested and ligated into the corresponding sites pGSA1403, The resulting construct 35S::pGSA1403MfbHLH38 was introduced into the A. tumefaciens strain LBA4404, and then transformed into Arabidopsis using the floral-dip transformation method [52]. $\mathrm{T}_{0}$ seeds were screened on $1 / 2$ MS medium supplying with kanamycin $(50 \mu \mathrm{g} / \mathrm{ml})$. The seedlings resistant to kanamycin were transplanted into pots with soil and the positive transgenic plants were further verified by PCR.
Three homozygous positive lines $\left(\mathrm{T}_{3}\right)$ were randomly selected for further experiments.

\section{Expression analysis of $\mathrm{MfbHLH} 38$ and ABA-responsive genes}

The four-week-old seedlings were subjected to normal condition, as well as simulated drought (10\% PEG-6000) and salt treatments $(300 \mathrm{mM} \mathrm{NaCl})$ for one day and four days, respectively. The leaves sampled from same positions of plants at one day and four days of treatments were used for expression level analysis. Total RNA extraction and the first-strand cDNA synthesis were conducted following the procedures mentioned above. The $25 \mu \mathrm{l}$ reaction mixture (Innovagene Biotech) was consisted of $12.5 \mu \mathrm{l}$ of $2 \times$ Taq SYBR Green qPCR Mix, $0.5 \mu \mathrm{l}$ of each primer $(10 \mu \mathrm{M}), 4 \mu \mathrm{l}$ of diluted $\mathrm{cDNA}$, and $7.5 \mu \mathrm{l}$ of Nuclease-free $\mathrm{H}_{2} \mathrm{O}$. The PCR was performed using CFX Connect fluorescent quantitative PCR instrument (Bio-Rad, Hercules, CA, USA), and the amplification condition was as follows: $94^{\circ} \mathrm{C}$ for $5 \mathrm{~min}, 42$ cycles of $94{ }^{\circ} \mathrm{C}$ for $8 \mathrm{~s}$, and $60^{\circ} \mathrm{C}$ for $60 \mathrm{~s}$. The AtActin 2 was used as the internal reference and the relative expression levels was calculated with $2^{-\Delta \Delta C T}$ method. Each qRT-PCR experiment performed as at least three technical and biological replicates. The primers used were for $M f b H L H 38$, 5' - TCGGAGAGAGGAAAACAAGC - 3' (forward) and $5^{\prime}$ - TTTTCCTTCACCCCAGACAC - 3' (reverse); NCED3, 5' - CGAGCCGTGGCCTAAAGTCT - 3' (forward) and 5' - GCTCCGATGAATGTACCGTGAA - 3' (reverse); P5CS, 5' - GGTGGACCAAGGGCAAGTAAGA TA - 3' (forward) and 5' - TCGGAAACCATCTGAGAA TCTTGT - 3' (reverse); RD29A, 5' - GATAACGTTGGA GGAAGAGTCGG - 3' (forward) and 5' - TCCTGATT CACCTGGAAATTTCG - 3' (reverse); and AtActin2, 5' GGAAGGATCTGTACGGTAAC - 3' (forward) and 5' TGTGAACGATTCCTGGACCT - 3' (reverse).

\section{Analysis of tolerance to drought and salinity}

For stress assays at seedling stage, the sterilized seeds were placed on $1 / 2 \mathrm{MS}$ solid medium varying in mannitol $(0-300 \mathrm{mM})$ and $\mathrm{NaCl}(0-150 \mathrm{mM})$ concentrations. Culture dishes were vertically settled and incubated with a cycle of $16 \mathrm{~h} / 8 \mathrm{~h}$ of light $\left(24^{\circ} \mathrm{C}\right) /$ dark $\left(22^{\circ} \mathrm{C}\right)$. Nine days later, the taproot length of each sample ( 15 seedlings per line for every petri dish) was measured. Each experiment was performed in three replicates.

To explore the drought and salinity tolerance for mature plants, the culture substrate after being fully infiltrated with water is equally divided into each pot. Approximately 50 vernalized $\left(4^{\circ} \mathrm{C}\right.$ for two days) seeds of each line were sown into pots under regular cultivation condition. Four-week-old plants were treated by drought and salinity stresses. For the drought treatment, every pot was firstly fully irrigated to ensure a saturated water 
content. For drought treatment, the watering was stopped immediately and continued for 15 days, and then rewatered. For salt treatment, plants were irregated with $\mathrm{NaCl}$ solution $(300 \mathrm{mM})$ twice at a 3-day interval. All plants were photographed every two or three days. Samples used for physiological index measurements were obtained through drying treatment (withhold watering) for 10 days and salt $(300 \mathrm{mM} \mathrm{NaCl})$ for seven days, respectively.

\section{Estimation of water loss rate}

Four-week-old plants were used for estimation of water loss rate. About $0.5 \mathrm{~g}$ leaves were excised from more than five plants in same status and put on filter paper on an experiment bench under condition of room temperature $\left(\sim 25^{\circ} \mathrm{C}\right)$ and $60 \%$ relative humidity. The leaves were weighted at designed time points. Water loss rate was then calculated and three replicates for each line were performed.

\section{Measurements of physiological index related to stress toleance}

Chlorophyll was extracted according to procedures described previously [53]. The modified acidic ninhydrin method was used to measure proline content [54]. The contents of soluble protein and soluble sugar were measured using TP Quantitative Assay Kit (Nanjing Jiancheng, Nanjing, China) and Plant Soluble Sugar Sontent Test Kit (Nanjing Jiancheng), respectively. Histochemical stains of $3,3^{\prime}$-diaminobenzidine (DAB) and nitroblue tetrazolium (NBT) was used to visualize the accumulated hydrogen peroxide $\left(\mathrm{H}_{2} \mathrm{O}_{2}\right)$ and superoxide anion radical $\left(\mathrm{O}_{2}{ }^{-}\right)$in leaves after stress treatment, respectively [50]. The $\mathrm{H}_{2} \mathrm{O}_{2}$ content as well as antisuperoxide anion activity were quantified by Hydrogen Peroxide assay kit (Nanjing Jiancheng) and Inhibition and Produce Superoxide Anion Assay Kit (Nanjing Jiancheng), respectively. Evaluation of superoxide dismutase (SOD), peroxidase (POD), and catalase (CAT) activities as well as malondialdehyde (MDA) content were conducted as described previously $[55,56]$. Three replicates were executed for all these experiments.

\section{Analysis of stomatal aperture and endogenous ABA content}

To measure stomatal movement responding to mannitol and ABA, rosette leaves of four-week-old WT and transgenic lines were floated on a solution $(50 \mathrm{mM} \mathrm{KCl}, 0.1$ $\mathrm{mM} \mathrm{CaCl}$, and $10 \mathrm{mM}$ MES, pH 6.15) and placed under light for $2.5 \mathrm{~h}$ to induce stomatal opening. Then, these leaves were transferred into the opening solutions without or with $300 \mathrm{mM}$ mannitol, or $20 \mu \mathrm{M}$ ABA, respectively, and treated in a growth incubator for a further $2 \mathrm{~h}$ [57-59]. Stomata on lower epidermal layers of the leaf were immediately observed and photographed by optical microscopy (DP80, Olympus, Japan). The width and length and their ratio (stomatal aperture) of more than 60 stomata for each line were measured and calculated. All experiments were repeated in three times.

Endogenous ABA content was measured by MetWare (http://www.metware.cn/) with the AB Sciex QTRAP 6500 LC-MS/MS platform. Briefly, $50 \mathrm{mg}$ leaves of fourweek-old WT and transgenic plants were extracted with 1 $\mathrm{ml}$ formic acid/water/methanol (0.5:2:7.5, V/V/V). The extract liquid was evaporated to dry in nitrogen, reconstituted with $0.1 \mathrm{ml} 80 \%$ methanol $(\mathrm{V} / \mathrm{V})$, and then filtered for detection. Three biological replicates were conducted.

\section{Statistical analysis}

Data generated in the present study were analyzed by the Independent-Sample T-Test in SPSS 23.0, and showed as the mean \pm standard deviation (SD) of three replicates, and the significance of difference were showed as $*(P<0.05)$ and $* *(P<0.01)$.

\section{Abbreviations \\ TFs: Transcription factors; ABA: Abscisic acid; YFP: Yellow fluorescent protein; qRT-PCR: Quantitative real-time PCR; WT: Wild-type; MDA: Malondialdehyde; ROS: Reactive oxygen species; $\mathrm{H}_{2} \mathrm{O}_{2}$ : Hydrogen peroxide; $\mathrm{O}_{2}{ }^{-}$: superoxide anion radical; SOD: Superoxide dismutase; POD: Peroxidase; CAT: Catalase}

\section{Acknowledgments}

Not applicable.

\section{Authors' contributions}

Experimental concept and design, $\mathrm{ZH}$ and $\mathrm{CZJ}$; Funding acquisition, $\mathrm{ZH}$; Investigation, JRQ, ZH, XYX, WXX, JTW, JC, YX, LS, SZC, LXS; Data analysis: JRQ, $\mathrm{ZH}, \mathrm{XL}$, JM Resources, CZ; Project administration and Supervision, ZH; Manuscript drafting, JRQ; Manuscript revision: JRQ, ZH, CZJ. All authors approved the final manuscript.

\section{Funding}

This research was supported by International Cooperation Project (2018H 0078 ) funded by Science and Technology Department of Sichuan Province, China, and Shuangzhi Plan funded by Sichuan Agricultural University. The funders had no role in the study design, collection, analysis, and interpretation of data, or in the writing of the report or decision to submit the article for publication.

\section{Availability of data and materials}

The sequence of MfbLHL38 has been deposited in GenBank of NCBI with accession No. of MT383747 (https://www.ncbi.nlm.nih.gov/nuccore/MT38374 7.1/). All other data generated or analyzed during this study are included in this published article.

Ethics approval and consent to participate

Not applicable.

Consent for publication

Not applicable.

\section{Competing interests}

The authors declare that they have no competing interests.

\section{Author details}

${ }^{1}$ College of Landscape Architecture, Sichuan Agricultural University, Wenjiang 611130, Sichuan, China. ${ }^{2}$ Department of Plant Sciences, University of California Davis, Davis, CA 95616, USA. ${ }^{3}$ Crops Pathology and Genetics 
Research Unit, United States Department of Agriculture, Agricultural Research Service, Davis, CA 95616, USA.

\section{Received: 4 April 2020 Accepted: 9 November 2020 Published online: 02 December 2020}

\section{References}

1. Vinocur B, Altman A. Recent advances in engineering plant tolerance to abiotic stress: achievements and limitations. Curr Opin Biotechnol. 2005;16:123-32.

2. Raghavendra AS, Gonugunta VK, Christmann A, Grill E. ABA perception and signalling. Trends Plant Sci. 2010;15:395-401.

3. de Zelicourt A, Colcombet J, Hirt H. The role of MAPK modules and ABA during abiotic stress signaling. Trends Plant Sci. 2016;21:677-85.

4. Mehrotra R, Bhalothia P, Bansal P, Basantani MK, Bharti V, Mehrotra S. Abscisic acid and abiotic stress tolerance - different tiers of regulation. J Plant Physiol. 2014;171:486-96.

5. Agarwal PK, Jha B. Transcription factors in plants and ABA dependent and independent abiotic stress signalling. Biol Plant. 2010;54:201-12.

6. Vishal B, Kumar PP. Regulation of seed germination and abiotic stresses by gibberellins and Abscisic acid. Front Plant Sci. 2018;9. https://doi.org/10. 3389/fpls.2018.00838.

7. Pires N, Dolan L. Origin and diversification of basic-helix-loop-helix proteins in plants. Mol Biol Evol. 2010;27:862-74.

8. Ludwig SR, Habera LF, Dellaporta SL, Wessler SR. LC, a member of the maize $R$ gene family responsible for tissue-specific anthocyanin production, encodes a protein similar to transcriptional activators and contains the mychomology region. PNAS. 1989;86:7092-6.

9. Bailey PC, Martin C, Toledo-Ortiz G, Quail PH, Huq E, Heim MA, et al. Update on the basic helix-loop-helix transcription factor gene family in Arabidopsis thaliana. Plant Cell. 2003;15:2497-502.

10. Carretero-Paulet L, Galstyan A, Roig-Villanova I, Martínez-García JF, BilbaoCastro JR, Robertson DL. Genome-wide classification and evolutionary analysis of the bHLH family of transcription factors in Arabidopsis, poplar, Rice, Moss, and Algae1[W]. Plant Physiol. 2010;153:1398-412.

11. Atchley WR, Terhalle W, Dress A. Positional dependence, cliques, and predictive motifs in the bHLH protein domain. J Mol Evol. 1999;48:501-16.

12. Nesi N, Debeaujon I, Jond C, Pelletier G, Caboche M, Lepiniec L. The TT8 gene encodes a basic helix-loop-helix domain protein required for expression of DFR and BAN genes in Arabidopsis Siliques. Plant Cell. 2000;12:1863-78.

13. Toledo-Ortiz G, Huq E, Quail PH. The Arabidopsis basic/helix-loop-helix transcription factor family. Plant Cell. 2003;15:1749-70.

14. Gao C, Sun J, Wang C, Dong Y, Xiao S, Wang X, et al. Genome-wide analysis of basic/helix-loop-helix gene family in peanut and assessment of its roles in pod development. PLoS One. 2017;12. https://doi.org/10.1371/journal. pone. 0181843

15. Jiang $Y$, Yang B, Deyholos MK. Functional characterization of the Arabidopsis bHLH92 transcription factor in abiotic stress. Mol Gen Genomics. 2009;282:503-16.

16. Liu W, Tai H, Li S, Gao W, Zhao M, Xie C, et al. bHLH122 is important for drought and osmotic stress resistance in Arabidopsis and in the repression of ABA catabolism. New Phytol. 2014;201:1192-204.

17. Babitha KC, Ramu SV, Pruthvi V, Mahesh P, Nataraja KN, Udayakumar M. Coexpression of AtbHLH17 and WRKY28 confers resistance to abiotic stress in Arabidopsis. Transgenic Res. 2013:22:327-41.

18. Wang F, Zhu H, Chen D, Li Z, Peng R, Yao Q. A grape bHLH transcription factor gene, $\mathrm{VvbHLH1}$, increases the accumulation of flavonoids and enhances salt and drought tolerance in transgenic Arabidopsis thaliana. Plant Cell Tissue Organ Cult. 2016;125:387-98.

19. Zhao M, Song A, Li P, Chen S, Jiang J, Chen F. A bHLH transcription factor regulates iron intake under Fe deficiency in chrysanthemum. Sci Rep. 2014; 4:6694.

20. Cui Y, Chen C-L, Cui M, Zhou W-J, Wu H-L, Ling H-Q. Four IVa bHLH transcription factors are novel Interactors of FIT and mediate JA inhibition of Iron uptake in Arabidopsis. Mol Plant. 2018;11:1166-83.

21. Abe H, Urao T, Ito T, Seki M, Shinozaki K, Yamaguchi-Shinozaki K. Arabidopsis AtMYC2 (bHLH) and AtMYB2 (MYB) function as transcriptional activators in Abscisic acid signaling. Plant Cell. 2003;15:63-78.

22. Li H, Wang L, Yang ZM. Co-expression analysis reveals a group of genes potentially involved in regulation of plant response to iron-deficiency. Gene. 2015;554:16-24
23. Kurt F, Filiz E. Genome-wide and comparative analysis of bHLH38, bHLH39, bHLH100 and bHLH101 genes in Arabidopsis, tomato, rice, soybean and maize: insights into iron (Fe) homeostasis. Biometals. 2018;31:489-504.

24. Moore JP, Farrant JM, Lindsey GG, Brandt WF. The south African and Namibian populations of the resurrection plant Myrothamnus flabellifolius are genetically distinct and display variation in their Galloylquinic acid composition. J Chem Ecol. 2005;31:2823-34.

25. Moore JP, Lindsey GG, Farrant JM, Brandt WF. An overview of the biology of the desiccation-tolerant resurrection plant Myrothamnus flabellifolia. Ann Bot. 2007;99:211-7.

26. Farrant JM. A comparison of mechanisms of desiccation tolerance among three angiosperm resurrection plant species. Plant Ecol. 2000;151:29-39.

27. Moore JP, Nguema-Ona E, Chevalier L, Lindsey GG, Brandt WF, Lerouge P, et al. Response of the leaf Cell Wall to desiccation in the resurrection plant Myrothamnus flabellifolius. Plant Physiol. 2006;141:651-62.

28. Drennan PM, Goldsworthy D, Buswell A. Marginal and laminar hydathodelike structures in the leaves of the desiccation-tolerant angiosperm Myrothamnus flabellifolius Welw. Flora Morphol Distrib Funct Ecol Plants. 2009;204:210-9.

29. Ma C, Wang H, Macnish AJ, Estrada-Melo AC, Lin J, Chang Y, et al. Transcriptomic analysis reveals numerous diverse protein kinases and transcription factors involved in desiccation tolerance in the resurrection plant Myrothamnus flabellifolia. Hortic Res. 2015;2:1-12.

30. Zhang L, Cheng J, Sun X, Zhao T, Li M, Wang Q, et al. Overexpression of VaWRKY14 increases drought tolerance in Arabidopsis by modulating the expression of stress-related genes. Plant Cell Rep. 2018;37:1159-72.

31. Marasco R, Rolli E, Ettoumi B, Vigani G, Mapelli F, Borin S, et al. A drought resistance-promoting microbiome is selected by root system under desert farming. PLoS One. 2012;7. https://doi.org/10.1371/journal.pone.0048479.

32. Dong Y, Wang C, Han X, Tang S, Liu S, Xia X, et al. A novel bHLH transcription factor PebHLH35 from Populus euphratica confers drought tolerance through regulating stomatal development, photosynthesis and growth in Arabidopsis. Biochem Biophys Res Commun. 2014;450:453-8.

33. Gururani M, Venkatesh J, Tran L-S. Regulation of photosynthesis during abiotic stress-induced Photoinhibition. Mol Plant. 2015;8:1304-20.

34. Ashraf M, Foolad MR. Roles of glycine betaine and proline in improving plant abiotic stress resistance. Environ Exp Bot. 2007;59:206-16.

35. Yang T, Yao S, Hao L, Zhao Y, Lu W, Xiao K. Wheat bHLH-type transcription factor gene TabHLH1 is crucial in mediating osmotic stresses tolerance through modulating largely the ABA-associated pathway. Plant Cell Rep. 2016:35:2309-23.

36. Davey M, Stals E, Panis B, Keulemans J, Swennen R. High-throughput determination of malondialdehyde in plant tissues. Anal Biochem. 2006:347:201-7.

37. Krasensky J, Jonak C. Drought, salt, and temperature stress-induced metabolic rearrangements and regulatory networks. J Exp Bot. 2012;63:1593-608.

38. Bose J, Rodrigo-Moreno A, Shabala S. ROS homeostasis in halophytes in the context of salinity stress tolerance. J Exp Bot. 2014;65:1241-57.

39. Chawla S, Jain S, Jain V. Salinity induced oxidative stress and antioxidant system in salt-tolerant and salt-sensitive cultivars of rice ( $O$ ryza sativa L.). J Plant Biochem Biotechnol. 2013;22:27-34.

40. Cutler SR, Rodriguez PL, Finkelstein RR, Abrams SR. Abscisic acid: emergence of a Core signaling network. Annu Rev Plant Biol. 2010;61:651-79.

41. Wang $\mathrm{H}-\mathrm{Y}$, Klatte $\mathrm{M}$, Jakoby $\mathrm{M}$, Bäumlein $\mathrm{H}$, Weisshaar B, Bauer P. Iron deficiency-mediated stress regulation of four subgroup $\mathrm{Ib} B H L H$ genes in Arabidopsis thaliana. Planta. 2007;226:897-908.

42. Tripathi DK, Singh S, Gaur S, Singh S, Yadav V, Liu S, et al. Acquisition and homeostasis of Iron in higher plants and their probable role in abiotic stress tolerance. Front Environ Sci. 2018;5. https://doi.org/10. 3389/fenvs.2017.00086.

43. Scandalios JG. Response of Plant Antioxidant Defense Genes to Environmental Stress. In: Scandalios JG, editor. Advances in Genetics: Academic Press; 1990. p. 1-41. https://doi.org/10.1016/S0065-2660(08)60522-2.

44. Gunes A, Cicek N, Inal A, Alpaslan M, Eraslan F, Guneri E, et al. Genotypic response of chickpea (Cicer arietinum L.) cultivars to drought stress implemented at pre- and post-anthesis stages and its relations with nutrient uptake and efficiency\&nbsp. Plant Soil Environ. 2011;52(8):368-76.

45. Samarah N, Mullen $\mathrm{R}$, Cianzio S. Size distribution and mineral nutrients of soybean seeds in response to drought stress. J Plant Nutr. 2004:27:815-35.

46. Zhu J-K. Plant salt tolerance. Trends Plant Sci. 2001;6:66-71.

47. Xu WF, Shi WM. Expression profiling of the 14-3-3 gene family in response to salt stress and potassium and Iron deficiencies in young tomato 
(Solanum lycopersicum) roots: analysis by real-time RT-PCR. Ann Bot. 2006; 98:965-74.

48. Pushnik JC, Miller GW, Manwaring JH. The role of iron in higher plant chlorophyll biosynthesis, maintenance and chloroplast biogenesis. J Plant Nutr. 1984;7:733-58.

49. Babaeian M, Piri I, Tavassoli A, Esmaeilian Y, Gholami H. Effect of water stress and micronutrients ( $\mathrm{Fe}, \mathrm{Zn}$ and $\mathrm{Mn}$ ) on chlorophyll fluorescence, leaf chlorophyll content and sunflower nutrient uptake in Sistan region. Afr J Agric Res. 2011;6:3526-31.

50. Fryer MJ, Oxborough K, Mullineaux PM, Baker NR. Imaging of photooxidative stress responses in leaves. J Exp Bot. 2002;53:1249-54.

51. Kumar S, Stecher G, Tamura K. MEGA7: molecular evolutionary genetics analysis version 7.0 for bigger datasets. Mol Biol Evol. 2016;33:1870-4.

52. Clough SJ, Bent AF. Floral dip: a simplified method for agrobacterium -mediated transformation of Arabidopsis thaliana. Plant J. 1998;16:735-43.

53. Palta JP. Leaf chlorophyll content. Remote Sens Rev. 1990;5:207-13.

54. Bates $L S$, Waldren RP, Teare ID. Rapid determination of free proline for water-stress studies. Plant Soil. 1973;39:205-7.

55. Zheng X, Tian S, Meng X, Li B. Physiological and biochemical responses in peach fruit to oxalic acid treatment during storage at room temperature. Food Chem. 2007;104:156-62.

56. Zhanyuan D, Bramlage WJ. Modified thiobarbituric acid assay for measuring lipid oxidation in sugar-rich plant tissue extracts. J Agric Food Chem. 1992; 40:1566-70.

57. Zhao Y, Liu M, He L, Li X, Wang F, Yan B, et al. A cytosolic NAD+-dependent GPDH from maize ( $\mathrm{ZmGPDH} 1$ ) is involved in conferring salt and osmotic stress tolerance. BMC Plant Biol. 2019;19:16.

58. Lim CW, Park C, Kim J-H, Joo H, Hong E, Lee SC. Pepper CaREL1, a ubiquitin E3 ligase, regulates drought tolerance via the ABA-signalling pathway. Sci Rep. 2017;7:477.

59. Jiang S-C, Mei C, Liang S, Yu Y-T, Lu K, Wu Z, et al. Crucial roles of the pentatricopeptide repeat protein SOAR1 in Arabidopsis response to drought, salt and cold stresses. Plant Mol Biol. 2015;88:369-85.

\section{Publisher's Note}

Springer Nature remains neutral with regard to jurisdictional claims in published maps and institutional affiliations.

Ready to submit your research? Choose BMC and benefit from:

- fast, convenient online submission

- thorough peer review by experienced researchers in your field

- rapid publication on acceptance

- support for research data, including large and complex data types

- gold Open Access which fosters wider collaboration and increased citations

- maximum visibility for your research: over $100 \mathrm{M}$ website views per year

At $\mathrm{BMC}$, research is always in progress.

Learn more biomedcentral.com/submissions 